\title{
A NOTE ON THE ESTIMATION OF MISSING PAIRWISE PREFERENCE VALUES: A UNINORM CONSISTENCY BASED METHOD*
}

\author{
FRANCISCO CHICLANA \\ Centre for Computational Intelligence, De Montfort University \\ Leicester LE1 $9 B \mathrm{BH}-U K$ \\ chiclana@dmu.ac.uk \\ ENRIQUE HERRERA-VIEDMA \\ Dept. of Computer Science and Artificial Intelligence, University of Granada \\ 18071 Granada - Spain \\ viedma@decsai.ugr.es \\ SERGIO ALONSO \\ Dept. of Software Engineering, University of Granada \\ 18071 Granada - Spain \\ zerjioi@ugr.es \\ FRANCISCO HERRERA \\ Dept. of Computer Science and Artificial Intelligence, University of Granada \\ 18071 Granada - Spain \\ herrera@decsai.ugr.es \\ Received 01 December 2007 \\ Revised 12 April 2008 \\ Accepted 10 July 2008
}

\begin{abstract}
Dealing with incomplete information is an important problem in decision making. In this paper, we present a short discussion on this topic and a new estimation method of missing values in an incomplete fuzzy preference relation which is based on the modelling of consistency of preferences via a representable uninorm.

Keywords: incomplete information, preferences, incomparability, consistency, transitivity, uninorms
\end{abstract}

\section{Introduction}

In decision making, there are cases where an expert would not be able to efficiently express any kind of preference degree between two or more of the available options. This may be

*Cite as: F. Chiclana, E. Herrera-Viedma, S. Alonso, F. Herrera: A Note on the Estimation of Missing Pairwise Preference Values: A Uninorm Consistency Based Method. Int. J. of Unc. Fuzziness and Knowledge Based Systems 16(supp 02), 19-32, August 2008. doi:10.1142/S0218488508005467 
due to an expert not possessing a precise or sufficient level of knowledge of part of the problem, or because that expert is unable to discriminate the degree to which some options are better than others. Sometimes, preferences with missing values are discarded, ${ }^{1}$ and when not they are penalised and rated more negatively than when complete preferences are provided. $^{2}$

In the last decade, many researchers have focused on developing methods to deal with problems with incomplete or incompletely identified information. One approach, in the literature of preference modelling, to tackle this problem is that of 'estimating' the missing pairwise preference values from the known ones using consistency criteria. ${ }^{3,4,5,6,7}$ Obviously, the main aim in the design of these type of procedures is to maintain or maximise the expert's global consistency. In this paper, we review shortly the problem of estimating missing values in decision making and present a new estimation method for incomplete fuzzy preference relations which is based on the modelling of consistency of preferences via a self-dual almost continuous uninorm operator, ${ }^{8}$ i.e. a representable uninorm operator.

The rest of the paper is set out as follows. Section 2 comprises a short review of different approaches to the problem of missing values in an expert's incomplete preference relation. Section 3 provides the necessary preliminaries on consistency of preferences, as well as the uninorm characterisation of consistency used for the introduction, in Section 4, of a new uninorm consistency based estimation procedure of unknown values in a fuzzy preference relation. Finally, conclusions are drawn in Section 5.

\section{Missing Pairwise Preference Values: A Short Discussion in Retrospect}

In Ref. 1, Millet compared different alternative preference elicitation methods and concluded that pairwise comparison methods were more accurate than non-pairwise methods. The main advantage of pairwise comparison is that of focusing exclusively on two alternatives at a time which facilitates experts when expressing their preferences. However, this way of providing preferences limits experts in their global perception of the alternatives and, as a consequence, the provided preferences could be inconsistent.

Given two alternatives, an expert might judge them in one of the following ways: (i) one alternative is preferred to another; (ii) the two alternatives are indifferent to him/her; (iii) he/she is unable to compare them. Fishburn pointed out that indifference might arise in three different ways: ${ }^{9}$

(a) when an expert truly feels that there is no real difference, in a preference sense, between the alternatives;

(b) when the expert is uncertain as to his/her preference between the alternatives because 'he might find their comparison difficult and may decline to commit himself[/herself] to a strict preference judgement while not being sure that he[/she] regards [them] equally desirable (or undesirable)';

(c) or when both alternatives are considered incomparable on a preference basis by the expert.

Therefore, incomparability and indifference are equivalent concepts for Fishburn. However, 
we believe that when an expert is unable to compare two alternatives then this situation should not be reflected in the preference relation as an indifference situation, but with a missing entry for that particular pair of alternatives. In other word, a missing value in a preference relation is not equivalent to a lack of preference of one alternative over another. A missing value might be also the result of the incapacity of experts to quantify the degree of preference of one alternative over another because "time pressure, lack of knowledge or data, and their limited expertise related to the problem domain," 10 in which case they may decide not to 'guess' to maintain the consistency of the values already provided. ${ }^{3}$

In a crisp context, the concept of consistency has traditionally been defined in terms of acyclicity, ${ }^{11,12}$ a condition closely related to the transitivity of preferences. However, the question whether the 'degree or strength of preference' of $x_{i}$ over $x_{j}$ exceeds, equals, or is less than the 'degree or strength of preference' of $x_{j}$ over $x_{k}$ cannot be answered by the classical preference modelling. The implementation of the degree of preference between alternatives may be essential in many situations, and this can be done by using fuzzy preference relations. ${ }^{13}$ In a fuzzy context, the traditional requirement to characterise consistency has followed the way of extending the classical requirements of binary preference relations. Thus, consistency is also based on the notion of transitivity. However, due to the role the intensity of preference has, we consider the term 'consistency' as described by Saaty in Ref. 14:

not merely the traditional requirement of the transitivity of preferences $[\ldots]$, but the actual intensity with which the preference is expressed transits through the sequence of objects in comparison.

It is quite often the case in empirical studies to discard a whole questionnaire when some data are missing. One example of this practice is reported by Millet in Ref. 1. However, Carmone et al. in Ref. 15 investigate the effect of reduce sets of pairwise comparisons. They compared results obtained for a complete pairwise comparison matrix and an incomplete one derived by eliminating known elements of the completed one. Their result suggests that 'random deletion of as much as $50 \%$ of the comparisons provide good results without compromising the accuracy.' However, because this process relies on the a priori knowledge of the completed pairwise comparison matrix it is therefore inapplicable in the real life applications. When a complete pairwise comparison matrix is not available, Carmone et. al suggest the selection of an appropriate methodology to 'build' the matrix.

A strong argument supporting this type of methodology is given by Ebenbach and Moore in Ref. 2: 'scenarios with missing values are normally penalised and rated more negatively than the same scenario with a value provided.' A system that helps experts to build a complete fuzzy preference relations in decision-making contexts has been developed in Ref. 16. This system reacts to an expert input of preference values by providing him/her recommendations on the preference values that he/she has not yet expressed.

In group decision-making, procedures that correct the lack of knowledge of a particular expert using the information provided by the rest of the experts together with some aggregation procedures can be found in. ${ }^{17,18}$ These approaches have several disadvantages. Among them we can cite: 
- The requirement of multiple experts in order to learn the missing value of a particular one.

- These procedures normally do not take into account the differences between experts' preferences, which could lead to the estimation of a missing value that would not naturally be compatible with the rest of the preference values given by that expert.

- Some of these missing information-retrieval procedures are interactive, that is, they need experts to collaborate in "real time", an option which is not always possible.

Different approaches to the above ones have been developed in. ${ }^{5,6,7}$ In these approaches, the estimation of missing values in an expert's incomplete preference relation is done using only the preference values provided by that particular expert. By doing this, it is assured that the reconstruction of the incomplete fuzzy preference relation is compatible with the rest of the information provided by that expert. Therefore, the main aim in the design of these procedures is to maintain or maximise the expert's global consistency.

Consistency is modelled in these studies via the additive transitivity property, which is equivalent to Saaty's consistency property for multiplicative preference relations. ${ }^{19,20,21,22}$ However, as we have shown in Ref. 23, this consistency property is in conflict with the corresponding scale used for providing the preference values.

In Ref. 8, a set of conditions was put forward for a fuzzy preference relation to be considered 'fully consistent.' Under this set of conditions it was shown that consistency of fuzzy preference relations can be characterised by representable uninorms. In the following section we provide a brief account on the necessary preliminaries on consistency of preferences, as well as a summary of its uninorm characterisation. This result is exploited in this paper for tackling the presence of incomparability in fuzzy preference relations. A uninorm consistency based method to estimate unknown values in the pair comparison of a set of alternatives based on the known ones is presented in section 4 .

\section{Reciprocal Fuzzy Preference Relations: Uninorm Based Consistency Property}

The introduction of the concept of 'fuzzy set' by Zadeh in Ref. 24 as an extension of the classical concept of set when applied to a binary relation leads to the concept of a fuzzy relation. Given a fuzzy preference relation $R=\left(r_{i j}\right)$ on a set of alternatives $X$, the following two semantics are found in the literature: ${ }^{25} r_{i j}$ may either reflect 'the intensity of preference (to what extent $x_{i}$ is preferred to $x_{j}$ )' or 'the uncertainty about preference (how sure it is that $x_{i}$ is preferred to $x_{j}$ )'. The fuzzy interpretation of intensity of preferences was introduced by Bezdek, Spillman and Spillman in Ref. 26 via the concept of a reciprocal fuzzy relation, and later reinterpreted by Nurmi in Ref. 27:

Definition 1. (Reciprocal Fuzzy Preference Relation) A reciprocal fuzzy preference relation $R$ on a finite set of alternatives $X$ is a fuzzy relation in $X \times X$ with membership function 
$\mu_{R}: X \times X \longrightarrow[0,1], \mu\left(x_{i}, x_{j}\right)=r_{i j}$, verifying

$$
r_{i j}+r_{j i}=1 \forall i, j \in\{1, \ldots, n\} .
$$

When cardinality of $X$ is small, the reciprocal fuzzy preference relation may be conveniently denoted by the matrix $R=\left(r_{i j}\right)$. The following interpretation is also usually assumed:

- $r_{i j}=1$ indicates the maximum degree of preference for $x_{i}$ over $x_{j}$.

- $\left.r_{i j} \in\right] 0.5,1\left[\right.$ indicates a definite preference for $x_{i}$ over $x_{j}$.

- $r_{i j}=0.5$ indicates indifference between $x_{i}$ and $x_{j}$.

\subsection{Transitivity and Consistency}

There are three fundamental and hierarchical levels of rationality assumptions when dealing with preference relations: ${ }^{28}$

- The first level of rationality requires indifference between any alternative $x_{i}$ and itself.

- The second one requires that if an expert prefers $x_{i}$ to $x_{j}$, that expert should not simultaneously prefer $x_{j}$ to $x_{i}$. This asymmetry condition is viewed as an "obvious" condition/criterion of consistency for preferences. ${ }^{9}$ This rationality condition is modelled by the property of reciprocity in the pairwise comparison between any two alternatives, ${ }^{29}$ which is seen by Saaty as basic in making paired comparisons. $^{14}$

- Finally, the third one is associated with the transitivity in the pairwise comparison among any three alternatives.

A preference relation verifying the third level of rationality is usually called a consistent preference relation and any property that guarantees the transitivity of the preferences is called a consistency property.

The value 0.5 is usually used to model the first level of rationality in the case fuzzy preference relations (definition 1): $r_{i i}=0.5(\forall i)$. The second level of rationality of fuzzy preferences is modelled using the aforementioned reciprocity property (definition 1): $r_{i j}+r_{j i}=1(\forall i, j)$. However, many properties or conditions have been suggested in the literature of fuzzy preferences to model the third level of rationality. Among these properties we can cite: (restricted) max-min transitivity, (restricted) max-max transitivity, additive transitivity, multiplicative transitivity. ${ }^{30,31}$ In Ref. 8, we have shown that under a set of conditions, multiplicative transitivity is, from the above list, the most appropriate one to model consistency of fuzzy preferences. The following subsection summarises this result.

\subsection{Uninorm Characterisation of Consistency}

The assumption of experts being able to quantify their preferences in the domain $[0,1]$ instead of $\{0,1\}$ underlies unlimited computational abilities and resources from the experts. 
Taking these unlimited computational abilities and resources into account, consistency of preferences we may formulated as follows:

$$
r_{i k}=f\left(r_{i j}, r_{j k}\right) \forall i, j, k
$$

being $f$ a function $f:[0,1] \times[0,1] \rightarrow[0,1]$.

In practical cases expression (1) might obviously not be verified even when the preference values of a preference relation are transitive. However, the assumption of modelling consistency using expression (1) can be exploited to estimate the missing values in an expert's incomplete fuzzy preference relation, using only the preference values provided by that particular expert, as shown in the following section.

The following properties are imposed to function $f$ :

Monotonicity: $f(x, y) \geq f\left(x^{\prime}, y^{\prime}\right)$ if $x \geq x^{\prime}$ and $y \geq y^{\prime}$

Associativity: $f(f(x, y), z)=f(x, f(y, z)) \forall x, y, z \in[0,1]$

Reciprocity: $f(x, y)+f(1-y, 1-x)=1 \quad \forall(x, y) \in[0,1] \times[0,1] \backslash\{(0,1),(1,0)\}$

Identity element: $f(0.5, x)=f(x, 0.5)=x \forall x \in[0,1]$

Continuity: $f$ is continuous in $[0,1] \times[0,1] \backslash\{(0,1),(1,0)\}$

Cancellative: $f(x, y)=f(x, z) \wedge f(y, x)=f(z, x) \forall x \in] 0,1[\Rightarrow y=z$

The following result was proved by Aczél in Ref. 32:

Theorem 1. Let I be a (closed, open, half-open, finite or infinite) proper interval of real numbers. Then $F: I^{2} \rightarrow I$ is a continuous operation on $I^{2}$ which satisfies the associativity equation

$$
F(F(x, y), z)=F(x, F(y, z)) \forall x, y, z \in I
$$

and is cancellative, that is,

$$
F\left(x_{1}, y\right)=F\left(x_{2}, y\right) \text { or } F\left(y, x_{1}\right)=F\left(y, x_{2}\right) \text { implies } x_{1}=x_{2} \text { for any } z \in I
$$

if, and only if, there exists a continuous and strictly monotonic function $\phi: J \longrightarrow I$ such that

$$
F(x, y)=\phi\left[\phi^{-1}(x)+\phi^{-1}(y)\right] \forall x, y \in I
$$

Here $J$ is one of the real intervals

$$
]-\infty, \gamma],]-\infty, \gamma[,[\delta, \infty[,] \delta, \infty[, \text { or }]-\infty, \infty[
$$

for some $\gamma \leq 0 \leq \delta$. Accordingly I has to be open at least from one side.

The function in (2) is unique up to a linear transformation of the variable $(\phi(x)$ may be replaced by $\phi(C x), C \neq 0$ but by no other function.)

The representation of function $F$ given by (2) coincides with Fodor, Yager and Rybalov representation theorem for almost continuous uninorms $U$, i.e. uninorms with identity element in $] 0,1\left[\right.$ continuous on $[0,1] \times[0,1] \backslash\{(0,1),(1,0)\} .{ }^{33}$ Therefore, the assumption of modelling consistency of reciprocal preferences in $[0,1]$ using the functional expression (1) has solution $f$ a representable uninorm operator with strong negator $N(x)=1-x$. $^{34}$ 
Following this result, we propose the following definition of consistent fuzzy reference relation:

Definition 2. Let $U$ be a representable uninorm operator with strong strong negator $N(x)=$ $1-x$. A fuzzy preference relation $R$ on a finite set of alternatives is consistent with respect to $U$ ( $U$-consistent) if

$$
\forall i, j, k:\left(r_{i k}, r_{k j}\right) \notin\{(0,1),(1,0)\} \Longrightarrow r_{i j}=U\left(r_{i k}, r_{k j}\right)
$$

Tanino's multiplicative transitivity property ${ }^{31}$ under reciprocity is the restriction to the region $[0,1] \times[0,1] \backslash\{(0,1),(1,0)\}$ of the following well known andlike representable uninorm: $:^{35}$

$$
U(x, y)=\left\{\begin{array}{l}
0, \quad(x, y) \in\{(0,1),(1,0)\} \\
\frac{x y}{x y+(1-x)(1-y)}, \text { otherwise }
\end{array}\right.
$$

The behaviour of uninorms on the squares $[0,0.5] \times[0,0.5]$ and $[0.5,1] \times[0.5,1]$ is closely related to t-norms and t-conorms. ${ }^{33}$ For the multiplicative uninorm (3), we have that

$$
U(x, y)=\frac{T_{U}(2 x, 2 y)}{2} \forall x, y \in[0,0.5]
$$

with

$$
T_{U}(x, y)=\frac{x y}{2-(x+y-x y)} \forall x, y \in[0,1]
$$

being the well known Einstein product.

\section{A U-Consistency Based Method to Estimate Missing Pairwise Preference Values}

Expression (1) can be used as a principle for deriving missing values. Indeed, using just those preference values provided by an expert, expression (1) could be used to estimate those preference values which were not given by that expert. By doing this, we assure that the estimated values are 'compatible' with the rest of the information provided by that expert. 5,36

\subsection{U-consistency Measure}

Given a fuzzy preference relation $R$ and $U$ a representable uninorm (with $N(x)=1-x$ ), the preference value $r_{i k}(i \neq k)$ can be partially $U$-estimated using an intermediate alternative $x_{j}$ such that $\left(r_{i j}, r_{j k}\right) \notin\{(0,1),(1,0)\}$ as follows

$$
u r_{i k}^{j}=U\left(r_{i j}, r_{j k}\right)
$$

The average of the partially $U$-estimated values obtained using all possible intermediate alternatives can be seen as the global consistency based estimated value

$$
u r_{i k}=\frac{\sum_{j \in R_{i k}^{01}} u r_{i k}^{j}}{\# R_{i j}^{01}}
$$


where

$$
R_{i k}^{01}=\left\{j \neq i, k \mid\left(r_{i j}, r_{j k}\right) \notin\{(0,1),(1,0)\}\right\}
$$

Example 1. For

$$
R=\left(\begin{array}{cccc}
0.5 & 0.55 & 0.7 & 0.95 \\
0.45 & 0.5 & 0.65 & 0.9 \\
0.3 & 0.35 & 0.5 & 0.75 \\
0.05 & 0.1 & 0.25 & 0.5
\end{array}\right)
$$

using the multiplicative uninorm (3) we get the following estimated preference values:

$$
U R=\left(\begin{array}{cccc}
0.5 & 0.62 & 0.78 & 0.9 \\
0.38 & 0.5 & 0.7 & 0.89 \\
0.22 & 0.3 & 0.5 & 0.86 \\
0.01 & 0.11 & 0.14 & 0.5
\end{array}\right)
$$

When the information provided in a fuzzy preference relation is completely $U$ consistent then $u r_{i k}^{j}$ coincides with $r_{i k}$ and we have $u r_{i k}=r_{i k}$. However, experts are not always fully consistent, and therefore the deviation of the actual value $r_{i k}$ with respect to its estimated one, $u r_{i k}$,

$$
\varepsilon u r_{i k}=\left|u r_{i k}-r_{i k}\right|
$$

can be used to measure the error in $[0,1]$ expressed in a preference degree between two alternatives with respect to $U$. Thus, it can be used to define the $U$-consistency level between the preference degree $r_{i k}$ and the rest of the preference values of the fuzzy preference relation:

Definition 3. Given $U$ a representable uninorm with $N(x)=1-x$, the $U$-consistency level associated with a preference value $r_{i k}$ is defined as

$$
U C L_{i k}=1-\varepsilon u r_{i k}
$$

When $U C L_{i k}=1$ then $\varepsilon u r_{i k}=0$ and there is no inconsistency at all. The lower the value of $U C L_{i k}$, the higher the value of $\varepsilon u r_{i k}$ and the more inconsistent is $r_{i k}$ with respect to the rest of information.

In the following, we define the $U$-consistency level of the whole reciprocal fuzzy preference relation $R$ :

Definition 4. Given $U$ a representable uninorm with $N(x)=1-x$, the $U$-consistency level of a fuzzy preference relation $R$ measured in $[0,1]$ is given as follows:

$$
U C L_{R}=\frac{\sum_{\substack{i, k=1 \\ i \neq k}}^{n} U C L_{i k}}{n(n-1)}
$$

Two considerations regarding the above definition of the consistency of a fuzzy preference relation. Firstly, the above definition of the consistency level has been given for a 
fuzzy preference relation which does not necessarily need to be reciprocal. If reciprocity holds then the above expression of the consistency level could be expressed as follows:

$$
U C L_{R}=\frac{2 \sum_{\substack{i, k=1 \\ i<k}}^{n} U C L_{i k}}{n(n-1)}
$$

Indeed, reciprocity of $R$ implies that $u r_{k i}=1-u r_{i k}$ because $R_{k i}^{01}=R_{i k}^{01}$ and $\forall j \in R_{k i}^{01}: u r_{k i}^{j}=U\left(r_{k j}, r_{j i}\right)=U\left(1-r_{j k}, 1-r_{i j}\right)=1-U\left(r_{j k}, r_{i j}\right)=1-U\left(r_{i j}, r_{j k}\right)=1-u r_{i k}^{j}$.

Therefore

$$
\varepsilon u r_{i k}=\left|u r_{i k}-r_{i k}\right|=\left|\left(1-u r_{k i}\right)-\left(1-r_{i k}\right)\right|=\varepsilon u r_{k i} .
$$

Consequently, for a reciprocal fuzzy reference relation $R, U C L_{i k}=U C L_{i k} \forall i, k$. Secondly, the $U$-consistency level is a well defined concept in the sense that a $U$-consistent fuzzy preference relation can be uniquely characterised by the above expression (8), as the following proposition states:

Proposition 1. A fuzzy preference relation $R$ is $U$-consistent if and if and only if $U C L_{R}=1$.

Proof. $U C L_{R}=1$ if and only if $\sum_{\substack{i, k=1 \\ i \neq k}}^{n} U C L_{i k}=n(n-1)$. Because $U C L_{i k} \in[0,1]$ then we have that $\sum_{\substack{i, k=1 \\ i \neq k}}^{n} U C L_{i k}=n(n-1)$ if and only if $U C L_{i k}=1 \forall i \neq k$. Thus, $U C L_{R}=1$ if and only if $u r_{i k}=r_{i k} \forall i \neq k$, which means that the preference relation $R$ is $U$-consistent.

Obviously, the lower $U C L_{R}$ the more inconsistent is $R$.

Example 2. For

$$
R=\left(\begin{array}{cccc}
0.5 & 0.55 & 0.7 & 0.95 \\
0.45 & 0.5 & 0.65 & 0.9 \\
0.3 & 0.35 & 0.5 & 0.75 \\
0.05 & 0.1 & 0.25 & 0.5
\end{array}\right)
$$

with the multiplicative uninorm we get:

$$
U C L=\left(\begin{array}{cccc}
1 & 0.93 & 0.92 & 0.95 \\
0.93 & 1 & 0.95 & 0.99 \\
0.92 & 0.95 & 1 & 0.89 \\
0.95 & 0.99 & 0.89 & 1
\end{array}\right)
$$

and

$$
U C L_{R}=0.94
$$

which means that $R$ is highly multiplicative consistent. 


\subsection{U-consistency Estimation of Missing Pairwise Preference Values}

Expression (5) needs to be extended to include the case when working with an incomplete fuzzy preference relation. To do this, the following sets are introduced:

$$
\begin{aligned}
A & =\{(i, k) \mid i, k \in\{1, \ldots, n\} \wedge i \neq k\} \\
M V & =\left\{(i, k) \in A \mid r_{i k} \wedge r_{k i} \text { are unknown }\right\} \\
E V & =A \backslash M V \\
H_{i k}^{01} & =\left\{j \in R_{i k}^{01} \mid(i, k) \in M V \wedge(i, j),(j, k) \in E V\right\}
\end{aligned}
$$

$M V$ is the set of pairs of alternatives for which the preference degree between them are unknown or missing; $E V$ is the set of pairs of alternatives for which the expert provides preference values; $H_{i k}^{01}$ is the set of intermediate alternatives $x_{j}(j \neq i, k)$ that can be used to estimate the unknown preference value $r_{i k}(i \neq k)$ via a representable uninorm (with $N(x)=1-x), U$. The final overall estimated preference value of a missing one, $u r_{i k}$, can be calculated when $\# H_{i k} \neq 0$, and will be defined as the average of the estimated values obtained using all the possible intermediate alternatives $x_{j}$

$$
u r_{i k}=\frac{\sum_{j \in H_{i k}^{01}} u r_{i k}^{j}}{\# H_{i k}^{01}} \text { if } \# H_{i k}^{01} \neq 0 .
$$

Note that when the preference relation is complete we have that $M V=\emptyset$ and $H_{i k}^{01}=R_{i k}^{01}$, with means that expression (9) is more general than (5).

An iterative procedure with the first task at each step of it being the identification of the missing preference values $r_{i k}(i \neq k)$ for which there exists at least one intermediate alternative $x_{j}$ that allows to apply expression (3) can be designed as in Ref. 5. The overall estimated values obtained at each step of this iterative procedure are added to the already known for the next step, with the procedure ending when no more missing values can be estimated. An incomplete fuzzy preference relation can be completed when a set of $n-1$ non leading diagonal preference vales, where each one of the alternatives is compared at least once, is known. The following example illustrates this iterative procedure:

Example 3. For

$$
R=\left(\begin{array}{cccc}
0.5 & 0.55 & - & - \\
0.45 & 0.5 & 0.65 & - \\
- & 0.35 & 0.5 & 0.75 \\
- & - & 0.25 & 0.5
\end{array}\right)
$$

using the multiplicative uninorm (3) and the known preference values, we can estimate in a first step the the missing preference values $r_{13}, r_{24}, r_{31}$ and $r_{42}$ :

$$
\begin{aligned}
& u r_{13}=u r_{13}^{2}=0.69 ; u r_{31}=u r_{31}^{2}=0.31 \\
& u r_{24}=u r_{24}^{3}=0.85 ; u r_{42}=u r_{42}^{3}=0.15
\end{aligned}
$$


After these missing values have been estimated, we have

$$
R=\left(\begin{array}{cccc}
0.5 & 0.55 & \mathbf{0 . 6 9} & - \\
0.45 & 0.5 & 0.65 & \mathbf{0 . 8 5} \\
\mathbf{0 . 3 1} & 0.35 & 0.5 & 0.75 \\
- & \mathbf{0 . 1 5} & 0.25 & 0.5
\end{array}\right)
$$

In a second (and final step) we can estimate the missing preference values $r_{14}, r_{41}$ :

$$
u r_{14}=\frac{u r_{14}^{2}+u r_{14}^{3}}{2}=0.87 ; u r_{41}=\frac{u r_{41}^{2}+u r_{41}^{3}}{2}=0.13
$$

and we obtained the following completed fuzzy preference relation

$$
R=\left(\begin{array}{cccc}
0.5 & 0.55 & 0.69 & \mathbf{0 . 8 7} \\
0.45 & 0.5 & 0.65 & 0.85 \\
0.31 & 0.35 & 0.5 & 0.75 \\
\mathbf{0 . 1 3} & 0.15 & 0.25 & 0.5
\end{array}\right)
$$

\section{Conclusions}

One approach, in the literature of preference modelling, to tackle the problem of missing data is that of 'estimating' the missing pairwise preference values from the known ones using consistency criteria. The consistency criteria used in reported procedures is modelled via the additive transitivity property. However, this consistency property is in conflict with the corresponding scale used for providing the preference values.

The assumption of experts being able to quantify their preferences in the domain $[0,1]$ instead of $\{0,1\}$ underlies unlimited computational abilities and resources from the experts. Taking these unlimited computational abilities and resources into account consistency of reciprocal fuzzy preference relations can be mathematically modelled via representable uninorms. This result, which in practical cases might obviously not be verified, has been exploited in this paper to design an iterative procedure to estimate missing preference values using only the rest of the preference values provided by a particular expert. Additionally, we have presented a short discussion on the problem of missing pairwise preference values.

\section{Acknowledgment}

This work has been supported by the EPSRC Research Grant EP/C542215/1, and the Research Projects TIN2007-61079, TIN2006-02121 and SAINFOWEB-PAI00602.

\section{References}

1. I. Millet, "The effectiveness of alternative preference elicitation methods in the analytic hierarchy process," Journal of Multi-Criteria Decision Analysis, vol. 6, pp. 41-51, 1997.

2. D. H. Ebenbach and C. F. Moore, "Incomplete information, inferences, and individual differences: the case of environmental judgements," Organizational Behavior and Human Decision Processes, vol. 81, pp. 1-27, 2000. 
3. S. Alonso, F. Chiclana, F. Herrera and E. Herrera-Viedma, "A learning procedure to estimate missing values in fuzzy preference relations based on additive consistency," Lecture Notes Artificial Intelligence, vol. 3131, pp. 227-238, 2004.

4. Z. Xu, "Goal programming models for obtaining the priority vector of incomplete fuzzy preference relation," International Journal of Approximate Reasoning, vol. 36, pp. 261-270, 2004.

5. E. Herrera-Viedma, F. Chiclana, F. Herrera and S. Alonso, "Group decision-making model with incomplete fuzzy preference relations based on additive consistency," IEEE Transactions on Systems, Man, Cybernetics - Part B: Cybernetics, vol. 37, pp. 176-189, 2007.

6. S. Alonso, F. Chiclana, F. Herrera, E. Herrera-Viedma, J.Alcalá-Fernández, and C. Porcel: "A consistency based procedure to estimate missing pairwise preference values," International Journal of Intelligent Systems, vol. 23, pp. 155-175, 2008.

7. M. Fedrizzi and S. Gioveb, "Incomplete pairwise comparison and consistency optimization," European Journal of Operational Research, vol. 183, pp. 303-313, 2007.

8. F. Chiclana, E. Herrera-Viedma, S. Alonso and F. Herrera, "Consistency of reciprocal preference relations," Proceedings of the 2007 IEEE International Conference on Fuzzy Systems, pp. 11191124, 2007.

9. P.C. Fishburn, Utility Theory for Decision Making, Robert E. Krieger Publishing Company, 1979.

10. S.H. Kim and B. S. Ahn, "Group decision making procedure considering preference strenght under incomplete information," Computers \& Operations Research, vol. 24, pp. 1101-1112, 1997.

11. F. Herrera, E. Herrera-Viedma and J.L. Verdegay, "A rational consensus model in group decision making using linguistic assessments,"Fuzzy Sets and Systems, vol. 88, pp. 31-49, 1997.

12. F.J. Montero and J. Tejada, "Some problems on the definition of fuzzy preference relations," Fuzzy Sets and Systems, vol. 20, pp. 45-53, 1986.

13. F. Chiclana, F. Herrera and E. Herrera-Viedma, "Integrating three representation models in fuzzy multipurpose decision making based on fuzzy preference relations," Fuzzy Sets and Systems, vol. 97, no. 1, pp. 33-48, 1998.

14. Th.L. Saaty, The Analytic Hierarchy Process, McGraw-Hill, New York, 1980.

15. F. J. Jr. Carmone, A. Kara and S. H. Zanakis, "A Monte Carlo investigation of incomplete pairwise comparison matrices in AHP," European Journal of Operational Research, vol. 102, 538533, 1997.

16. S. Alonso, F. J. Cabrerizo, F. Chiclana, F. Herrera, and E. Herrera-Viedma, "An interactive decision support system based on consistency criteria," Journal of Multiple-Valued Logic \& Soft Computing, vol. 14, pp. 371-386, 2008.

17. J. K. Kim, S. H. Choi, C. H. Han and S. H. Kim, "An interactive procedure for multiple criteria group decision making with incomplete information," Computers \& Industrial Engineering, vol. 35, pp. 295-298, 1998.

18. J. K. Kim and S. H. Choi, "A utility range-based interactive group support system for multiattribute decision making," Computers \& Operations Research, vol. 28, pp. 485-503, 2001.

19. F. Chiclana, F. Herrera and E. Herrera-Viedma, "Integrating multiplicative preference relations in a multipurpose decision making model based on fuzzy preference relations," Fuzzy Sets and Systems, vol. 122, pp. 277-291, 2001.

20. F. Chiclana, F. Herrera, and E. Herrera-Viedma. "A note on the internal consistency of various preference representations," Fuzzy Sets and Systems, vol. 131, pp. 75-78, 2002.

21. E. Herrera-Viedma, F. Herrera, F. Chiclana and M. Luque, "Some issues on consistency of fuzzy preference relations," European Journal of Operational Research, vol. 154, pp. 98-109, 2004.

22. F. Chiclana, F. Mata, S. Alonso, E. Herrera-Viedma and L. Martínez, "Integration of a consistency control module within a consensus decision making model," International Journal of Uncertainty, Fuzziness and Knowledge Based Systems, vol. 16, No. supp01, pp. 35-53, 2008. 
23. F. Chiclana, E. Herrera-Viedma, S. Alonso and R.A. Marqués Pereira, "Preferences and consistency issues in group decision making," In: H. Bustince, F. Herrera, J. Montero (editors): Fuzzy Sets and Their Extensions: Representation, Aggregation and Models, Studies in Fuzziness and Soft Computing, Springer Verlag, vol. 220, pp. 219-237, 2008.

24. L.A. Zadeh, "Fuzzy sets," Information and Control, vol. 8, pp. 338-353, 1965.

25. D. Dubois and H. Prade, "The three semantics of fuzzy sets," Fuzzy Sets and Systems, vol. 90, pp. 141-150, 1997.

26. J. Bezdek, B. Spillman and R. Spillman, "A fuzzy relation space for group decision theory," Fuzzy Sets and Systems, vol. 1, pp. 255-268, 1978.

27. H. Nurmi, "Approaches to collective decision making with fuzzy preference relations," Fuzzy Sets and Systems, vol. 6, pp. 249-259, 1981.

28. J.L. García-Lapresta and R.A. Marques Pereira, "The self-dual core and the anti-self-dual remainder of an aggregation operator," Fuzzy Sets and Systems, vol. 159, pp. 47-62, 2008.

29. F. Chiclana, F. Herrera, E. Herrera-Viedma and L. Martínez, "A note on the reciprocity in the aggregation of fuzzy preference relations using OWA operators," Fuzzy Sets and Systems, vol. 137, pp. 71-83, 2003.

30. D. Dubois and H. Prade, Fuzzy Sets and Systems: Theory and Application, Academic Press, New York, 1980.

31. T. Tanino, "Fuzzy preference relations in group decision making," in J. Kacprzyk and M. Roubens (Eds.) Non-Conventional Preference Relations in Decision Making, Springer-Verlag, Berlin, 1988, pp. 54-71.

32. J. Aczél, A Short Course on Functional Equations, D. Reidel Publishing Company, Dordrecht, 1987.

33. J.C. Fodor, R.R. Yager and A. Rybalov, "Structue of uninorms," International Journal of Uncertainty, Fuzziness and Knowledge-Based Systems, vol. 5, pp. 411-427, 1997.

34. R.R. Yager and A. Rybalov, "Uninorms aggretation operators," Fuzzy Sets and Systems, vol. 80, pp. 111-120, 1996.

35. E.P. Klement, R. Mesiar and E. Pap, "On the relationship of associative compensatory operators to triangular norms and conorms," International Journal of Uncertainty, Fuzziness and Knowledge-Based Systems, vol. 4, pp. 129-144, 1996.

36. E. Herrera-Viedma, S. Alonso, F. Chiclana and F. Herrera, "A consensus model for group decision making with incomplete fuzzy preference relations," IEEE Transactions on Fuzzy Systems, vol. 15 , pp. 863-877, 2007. 